\section{Antibiotic Intramedullary Nail in the Management of Infect- ed Ununited Tibial Fractures - Case Report, Technique and Review of Literature}

\section{John McMurtry* and Varatharaj Mounasamy}

Department of Orthopaedic Surgery, Virginia Commonwealth University Health System, USA

\begin{abstract}
Surgical management of an ununited tibial fracture with concomitant infection presents a challenging situation to the treating surgeon even with recent advances in antibiotic delivery, fracture fixation, soft tissue management, and wound care. This problem is often caused by bacteria capable of forming a biofilm on orthopedic implants which is an immunopriviledged site causing systemic antibiotics to be unsuccessful in providing a definitive solution. Antibiotic elution through the use of Poly Methyl Methacrylate (PMMA) in the form of an intramedullary tibial nail fills dead space, stabilizes factures, and delivers a high dose of antibiotics to the zone of infection. We present a series of three patients with traumatic infected nonunions of the tibia who were successfully treated with a custom-made antibiotic cement nail functioning.
\end{abstract}

\section{Introduction}

Surgical management of an ununited tibial fracture with concomitant infection presents a challenging situation to the treating surgeon even with recent advances in antibiotic delivery, fracture fixation, soft tissue management, and wound care. This complex patient presentation is twofold in that infection degrades the primary bone healing response leading to a pathologic rate of motion at the fracture site [1]. It is the dual threat, of infection and inadequate fixation, to bone healing that leads to the poor patient outcomes reported in infected nonunions. Two classical approaches have been advocated for treatment of infected nonunions with the "Conventional" treatment and a second "Active" approach [1]. The "Conventional" treatment focuses on normalization of the overlying soft tissue and cessation any continued draining prior to obtaining a bony union,

${ }^{*}$ Corresponding author: John McMurtry, Department of Orthopaedic Surgery, Virginia Commonwealth University, USA, Tel: +1 8048271204; Fax: +1 8048281572; E-mail: John.McMurtry@vcuhealth.org

Citation: McMurtry J, Mounasamy V (2016) Antibiotic Intramedullary Nail in the Management of Infected Ununited Tibial Fractures - Case Report, Technique and Review of Literature. J Clin Stud Med Case Rep 3: 031.

Received: February 27, 2016; Accepted: March 22, 2016; Published: April 06 2016 whereas the "Active" treatment attempts to expedite formation of a bony union with subsequent treatment of the infection [1]. In this same vein, Cierny and Mader put forth principals necessary to cure osteomyelitis. Successful management of osteomyelitis is predicated on meticulous debridement, dead space minimization, bony stabilization, soft tissue coverage, and optimum antibiotic delivery [2].

Orthopedic trauma with resultant infection is often caused by bacteria capable of forming a biofilm, which protects the microbe from the immune system by forming a protein and polysaccharide matrix [3]. This biofilm is resistant to systemic and local antibiotics in isolation, but combined with thorough debridement a cure is more likely. The administration of focused antibiotics to the specific organism or organisms is a mainstay in treatment, but systemic antibiotics have the limitation of toxicity to the human host. To increase the delivery of antibiotics to local tissues and to minimize systemic toxicity antibiotics were incorporated into Poly Methyl Methacrylate (PMMA) [4]. Buchholz and Engelbrecht pioneered the use of antibiotic cement for treatment of osteomyelitis and found antibiotic concentrations which were vastly higher and longer lasting than systemic or other local depot administration [5]. Bone cement loaded with antibiotics is easily customized to the size and shape necessary to function as an intramedullary nail, which fills dead space, stabilizes factures, and delivers a high dose of antibiotics to the zone of infection [6]. Paley published on a temporizing treatment for intramedullary infections after nailing which consisted of using a custom-made antibiotic impregnated cement rod which can be removed after 6 weeks and replaced with definitive metal intramedullary nail [7]. With the goal of curing localized osteomyelitis and maintaining fracture stability we presents a report of three patients with traumatic infected ununited fractures of the tibia treated with a custom-made antibiotic cement rod which functions as definitive treatment without the need for eventual replacement with a metal intramedullary nail.

\section{Surgical Technique}

The technique of Paley and Herzenberg was used for the treatment of all three patients with minimal substitutions and alterations to the protocol [7]. The intramedullary nail was removed with all locking screws and the medullary canal was reamed several millimeters greater than the previous nail diameter. The reamed bone and the site of the nonunion were thoroughly debrided and tissue sent for culture. The medullary canal and fracture site were copiously irrigated with normal saline without any additional antibiotic supplementation. The necessary materials are as follows: IM guide wire, $80 \mathrm{~g}$ gentamicin PMMA powder, $2 \mathrm{~g}$ vancomycin powder, sterile mineral oil, and a chest tube (Figure 1). The guidewire was measured to the length of the medullary canal and a chest tube of the approximate size of the removed nail was selected. The chest tube is cut to the measured medullary length, pretreated with mineral oil and the guidewire is cut $5 \mathrm{~mm}$ longer than measured and bent into a loop for the proximal portion of the nail (Figure 2). The antibiotic powder of 2 grams of vancomycin and PMMA cement is vacuum mixed with liquid monomer and injected into the chest tube. The precut guidewire is then inserted into the chest tube and once the cement begins to 
Citation: McMurtry J, Mounasamy V (2016) Antibiotic Intramedullary Nail in the Management of Infected Ununited Tibial Fractures - Case Report, Technique and Review of Literature. J Clin Stud Med Case Rep 3: 031.

- Page 2 of 5 •

harden the chest tube is cut longitudinally and removed from the nail to allow the monomer to evaporate (Figure 3). Care must be taken at this point not to peel the chest tube away too quickly as the uncured cement can peel away from the wire and remain attached to the plastic. Once the cement nail is fully cured and cooled in room air it is cleaned of any remaining debris or mineral oil. The cleaned and cured nail is then inserted into the medullary canal with the guidewire loop left protruding from the proximal canal. The wound was closed and if necessary soft tissue flaps were created to cover any identified defects preoperatively. All patients were placed into splints postoperatively for a minimum of 6 weeks to minimize motion at the fracture sites to maximize osseous healing.

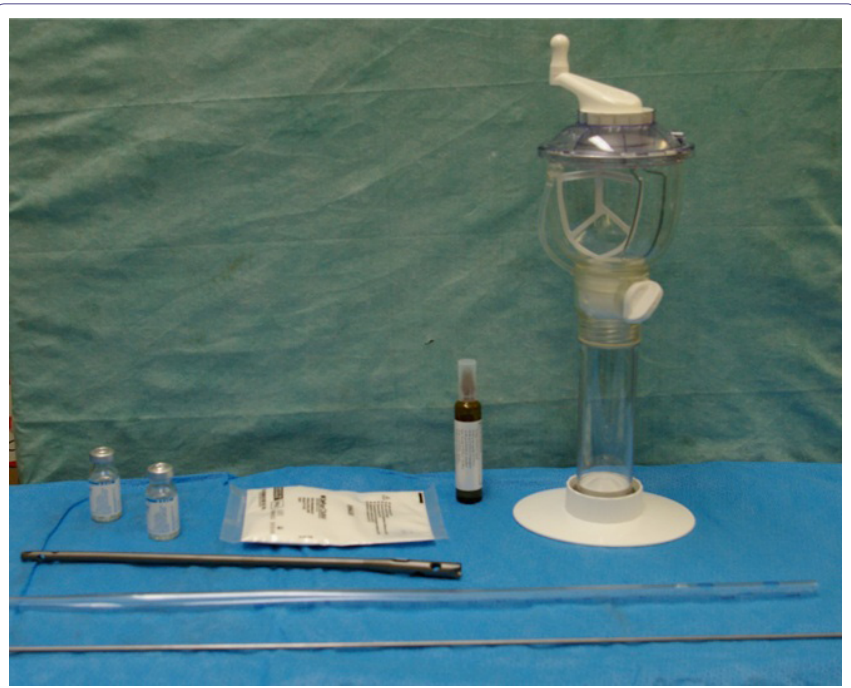

Figure 1: IM guide wire, $80 \mathrm{~g}$ gentamicin PMMA powder, $2 \mathrm{~g}$ vancomycin powder, sterile mineral oil, and a chest tube.

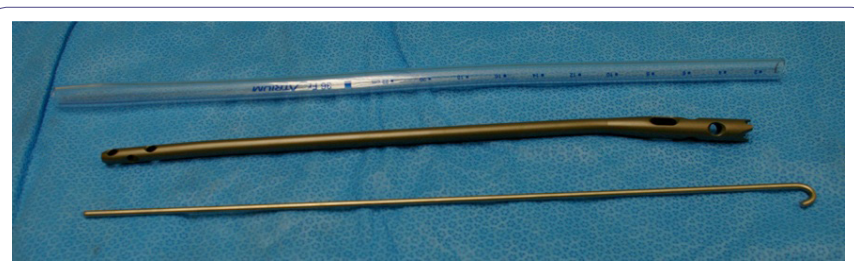

Figure 2: Chest tube cut to size, removed intramedullary nail, guide wire cut to size and bent at the end.

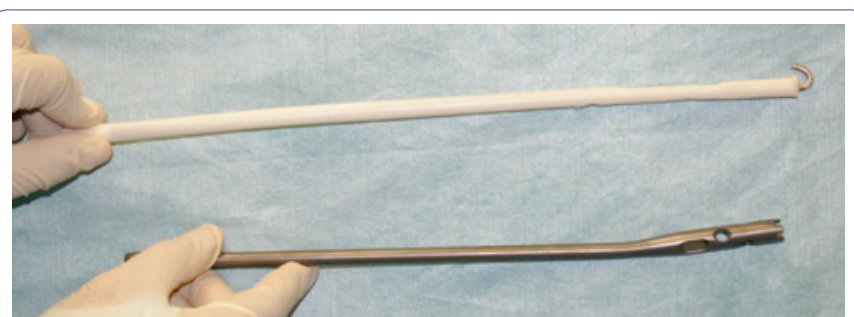

Figure 3: Final antibiotic nail of the approximate diameter and length of the removed intramedullary nail. Curved proximal wire to facilitate removal, insertion, and manipulation.

\section{Case 1 (Figure 4)}

A 22 year male was referred from an outside hospital to the orthopedic clinic 5 weeks status post intramedullary nailing of a grade II open fracture to the right tibia from a gunshot wound. The patient was found to have a draining wound over the site of the gunshot wound and the open fracture. The patient was not acutely ill or taking antibiotics at the time. The patient was taken to the operating room to undergo removal of the intramedullary nail, culture of intramedullary reamings, and placement of an antibiotic cement rod/spacer. The antibiotic spacer was placed using the surgical technique described in the text above. The patient was given piperacillin/tazobactam, and imipenem/cilastatin in a Peripherally Inserted Central Catheter (PICC) for positive cultures of Enterobacter and Peptostreptococcus. The surgical wounds healed without complication and the patient was advanced to full weight bearing 3 months after placement of the antibiotic cement nail once signs of radiographic union were identified. Bony union was achieved with non-weight bearing status, no bone grafting procedures, and no bone stimulator use. The antibiotic cement rod was left in place and the patient fully healed the fracture site without additional clinically significant deficit in the extremity.



\section{Case 2 (Figure 5)}

A 44 year male was referred from an outside hospital to the orthopedic clinic 12 weeks status post intramedullary nailing of a grade II open fracture to the right tibia from a motorcycle collision. The patient was found to have an unhealed draining wound over the site of the open fracture and had not yet been placed on antibiotics. The patient was not acutely ill, but was taken to the OR to undergo removal of the intramedullary nail, culture of intramedullary reamings, and placement of an antibiotic cement rod/spacer. The patient had infectious materials extending proximal and distal to the fracture site which required additional dissection. The antibiotic spacer was placed using the surgical technique described in the text 
Citation: McMurtry J, Mounasamy V (2016) Antibiotic Intramedullary Nail in the Management of Infected Ununited Tibial Fractures - Case Report, Technique and Review of Literature. J Clin Stud Med Case Rep 3: 031.

above. The patient was given a vancomycin, piperacillin/ tazobactam, and nafcillin in a Peripherally Inserted Central Catheter (PICC) for positive cultures of methicillin susceptible Staphylococcus aureus for 6 weeks post operatively. Postsurgical wounds healed without complication and splitting was maintained for six weeks. The patient was then advanced to full weight bearing after radiographic signs of union were observed at approximately 3 months post operatively. At final follow up the patient had completely healed the fracture without significant clinical deformity.
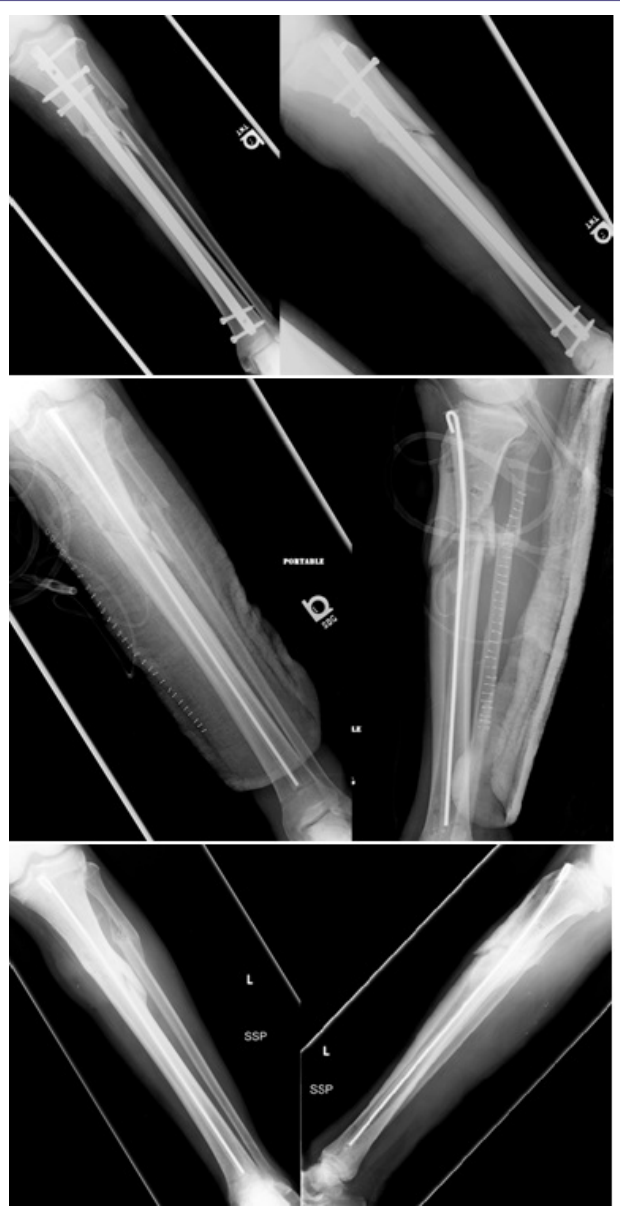

Figure 5: Case 2 presenting preoperative, postoperative, and final radiographs from placement of antibiotic cement intramedullary nail.

\section{Case 3 (Figure 6)}

A 75 year male patient was brought to our Level 1 trauma to undergo workup and treatment after being involved in a motor vehicle collision. The patient was wound to have a grade III open fracture of the left proximal third tibial diaphysis. The patient underwent administration of an intravenous first generation cephalosporin antibiotic every 8 hours after his initial dose in the emergency department until definitive fixation. The patient was unable to undergo fixation acutely with an intramedullary tibial nail secondary to trauma induced medical instability for several days. On Hospital day three the patient was taken to the operating room to undergo irrigation and debridement with placement of a tibial intramedullary nail. The patient's wounds were closed primarily and he was monitored closely while in the hospital. At the patients six week follow up appointment he was noted to have wound dehiscence with drainage. Antibiotics were withheld until the patient was taken to the OR to undergo removal of the intramedullary nail, culture of intramedullary reamings, placement of an antibiotic cement rod/spacer, and wound coverage with a medial gastrocnemius flap. The antibiotic spacer was placed using the surgical technique described in the text above. The patient was given a vancomycin and rifampin in a Peripherally Inserted Central Catheter (PICC) for positive cultures of methicillin resistant Staphylococcus aureus, corynebacter, Peptostreptococcus and Acinetobacter calcoaceticus - baumanii complex for 6 weeks post operatively. The surgical wounds healed and the patient was splinted for 8 weeks and then advanced to full weight bearing 3 months after placement of the antibiotic cement nail weight bearing was only begun after signs of radiographic union were identified on plain radiographs. As in the previous two cases bony union was achieved with non-weight bearing status, no bone grafting procedures, and no bone stimulator use. The antibiotic cement rod was left in place and the patient healed the fracture site without significant deformity or deficit in the extremity.
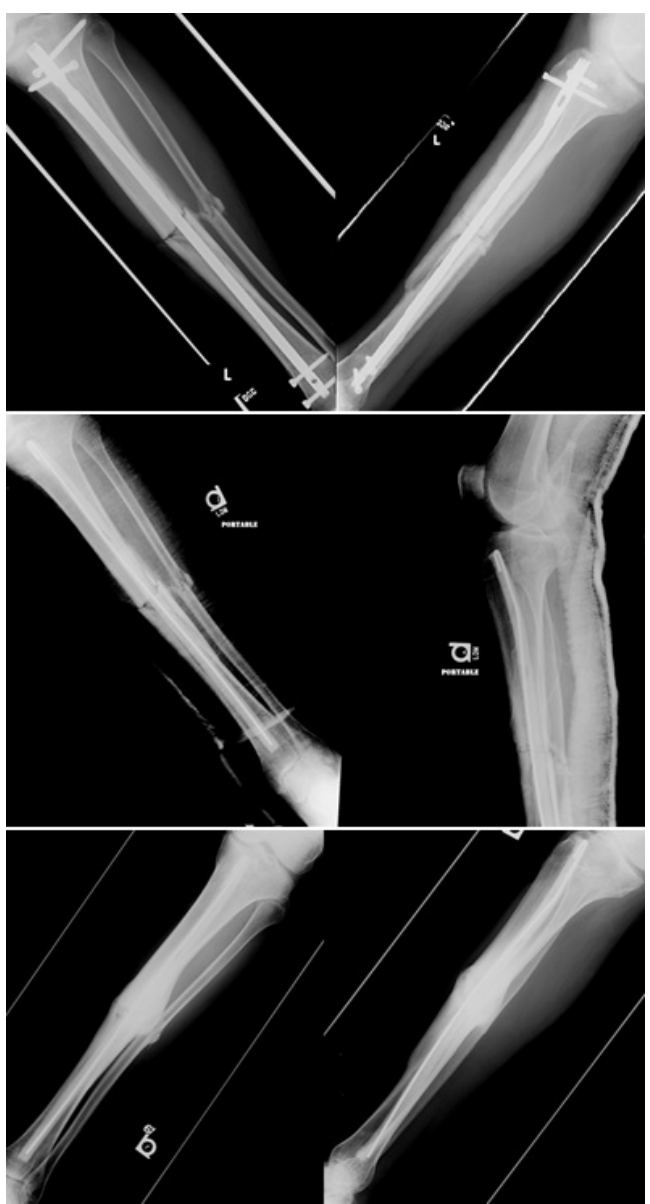

Figure 6: Case 3 presenting preoperative, postoperative, and final radiographs from placement of antibiotic cement intramedullary nail.

\section{Discussion}

Post traumatic infected ununited fractures still remain a challenge to the orthopedic surgeon due to the need to provide bony stabilization and to deliver high levels of antibiotics at the fracture site. Tibial fractures associated with significant soft tissue damage and disrupted vascularity are at increased risk of infection, nonunion, and other associated complications [8]. Within this setting, perioperative antibiotics are thought to decrease the chance of post-operative infection, but in the presence of surgical implants such as intramedullary nails they have limited efficacy [9]. To improve prophylaxis and 
treatment of infections related to orthopedic implants the delivery of local antibiotics using antibiotic coated implants represents an important treatment option $[10,11]$.

Multiple methods of local antibiotic delivery are in use today, but the most common is PMMA due its ability to achieve high concentrations in the local tissue and to avoid systemic toxicity. Initially, cases of intramedullary osteomyelitis were treated with reaming and external fixation. This advanced to reaming, external fixation, and placement of antibiotic beads to sterilize the canal prior to definitive fixation with metal intramedullary nail implant [12]. The use of antibiotic beads has been closely evaluated, and demonstrated successful clinical outcomes as evidenced by Ostermann. He obtained a threefold decrease in acute and local osteomyelitis with the use of local and systemic antibiotics compared to systemic antibiotics alone [13]. In an effort to further simplify the infection algorithm Fitzgerald attempted to treat chronic osteomyelitis with antibiotic beads alone without debridement, yet this did not demonstrate clinical efficacy [14]. Although the clinical use of antibiotic beads has been validated, the duration of use and the need for removal remain controversial. Some authors advocate for the removal of beads at 4 to 6 weeks post implantation due to concern over the beads acting as a foreign body, increasing antibiotic resistance by proving a constant sub-therapeutic antibiotic level, and increased difficulty in removal as beads become encapsulated by callous and fibrous tissue $[4,15]$. This limited time frame of efficacy is related to the exponential decline of antibiotic concentrations just one day after implantation leading to subtherapeutic levels 6 weeks post operatively $[16,17]$. The use of an antibiotic cement rod has the benefit of maintaining a smooth surface to facilitate removal any time point in the future, continued elution of therapeutic levels of antibiotics for several weeks, and maintenance of the intramedullary space necessary for final metal intramedullary nail implantation if desired $[7,18]$. There also exists a plethora of other treatment options to include external fixation, although many questions remain to be answered, our method of antibiotic-impregnated cement nails, based on the work by Paley and Herzenberg, as definitive treatment in infected ununited tibial fractures appears to give successful clinical results [7].

There are some limitations to the current technique which was elucidated by Shyam et al., when they demonstrated that Antibiotic coated intramedullary nail could not be used for definitive treatment when bone defects were greater than $6 \mathrm{~cm}$ [19]. These results correlate with the findings of Thonse and Conway who had difficulty achieving an uninfected union with large bone defects. Our patient population did not demonstrate any large defects and as such our technique achieved the desired outcome. Another limitation to our proposed treatment strategy is that we reflexively use gentamicin and vancomycin as our antibiotics of choice for all nails. In regards to selection of antibiotic, vancomycin and gentamicin are by far the most commonly used agents with minimal reports of systemic toxicity [20-22]. Gentamicin and vancomycin are broad spectrum antibiotics, stable for the exothermic reaction present in PMMA, work synergistically, and have been reported to increase elution rates when used simultaneously [21-24]. The optimal solution to antibiotic selection would be isolation of the offending organism and subsequent use of a targeted antibiotic mixed into the PMMA. This represents a dilemma as these wounds are often colonized with multiple organisms, pre procedure antibiotics diminish culture yields, and the causative organism is frequently not identified [5,21]. In order not to delay definitive treatment to obtain cultures which frequently will not assist in eradication of the infection we recommend the use of broad spectrum antibiotics and early surgical intervention. Additionally, no definitive evidence is present which attributes the eradication of infection and the creation of bony union to our antibiotic nail construct as opposed to it being a result of systemic antibiotics and replacement of an intramedullary implant. Our technique could be improved by cooling the curing cement in a cold sterile water basin to minimize melting of the chest tube and subsequent increased difficulty and time to prepare the final implant [25].

\section{References}

1. LaVelle DG (2003) Delayed union and nonunion of fractures. Campbell's operative orthopaedics 3: $2579-2629$.

2. Cierny G 3rd, Mader JT, Penninck JJ (2003) A clinical staging system for adult osteomyelitis. Clin Orthop Relat Res 414: 7-24.

3. Stoodley P, Ehrlich GD, Sedghizadeh PP, Hall-Stoodley L, Baratz ME, et al (2011) Orthopaedic biofilm infections. Curr Orthop Pract 22: 558-563.

4. Zalavras CG, Patzakis MJ, Holtom P (2004) Local antibiotic therapy in the treatment of open fractures and osteomyelitis. Clin Orthop Relat Res: 86-93.

5. Buchholz HW, Elson RA, Engelbrecht E, Lodenkämper H, Röttger J, et al. (1981) Management of deep infection of total hip replacement. J Bone Join Surg $\mathrm{Br}$ 63: 342-353.

6. Wasko MK, Kaminski R (2015) Custom-Made Antibiotic Cement Nails in Orthopaedic Trauma: Review of Outcomes, New Approaches, and Perspectives. BioMed Research International.

7. Paley D, Herzenberg JE (2002) Intramedullary infections treated with antibiotic cement rods: preliminary results in nine cases. J Orthop Trauma 16 723-729.

8. Gaebler C, Berger U, Schandelmaier P, Greitbauer M, Schauwecker HH, et al. (2001) Rates and odds ratios for complications in closed and open tibial fractures treated with unreamed, small diameter tibial nails: a multicenter analysis of 467 cases. J Orthop Trauma 15: 415-423.

9. Diefenbeck M, Mückley T, Hofmann GO (2006) Prophylaxis and treatment of implant-related infections by local application of antibiotics. Injury 2: 95-104.

10. Mendelsohn ES, Warganich T, Nielsen ES, Najibi S (2013) Application of an antibiotic intramedullary nail in the management of a large metacarpal bone defect. Tech Hand Up Extrem Surg 17: 187-191.

11. Fuchs $T$, Stange R, Schmidmaier G, Raschke MJ (2011) The use of gentamicin-coated nails in the tibia: preliminary results of a prospective study. Arch Orthop Trauma Surg 131: 1419-1425.

12. Thonse R, Conway J (2007) Antibiotic cement-coated interlocking nail for the treatment of infected nonunions and segmental bone defects. J Orthop Trauma 21: 258-268.

13. Ostermann PA, Seligson D, Henry SL (1995) Local antibiotic therapy for severe open fractures. A review of 1085 consecutive cases. J Bone Joint Surg $\mathrm{Br}$ 77: 93-97.

14. Fitzgerald RH (1983) Experimental osteomyelitis: description of a canine model and the role of depot administration of antibiotics in the prevention and treatment of sepsis. J Bone Joint Surg Am 65: 371-380.

15. Salvati EA, Callaghan JJ, Brause BD, Klein RF, Small RD (1986) Reimplantation in infection. Elution of gentamicin from cement and beads. Clin Orthop Relat Res : 83-93.

16. Meyer J, Piller G, Spiegel CA, Hetzel S, Squire M (2011). Vacuum-mixing significantly changes antibiotic elution characteristics of commercially available antibiotic-impregnated bone cements. J Bone Joint Surg Am 93: 2049-2056.

17. Passuti N, Gouin F (2003) Antibiotic-loaded bone cement in orthopedic surgery. Joint Bone Spine 70: 169-174. 

and Review of Literature. J Clin Stud Med Case Rep 3: 031.

18. Nelson CL, Hickmon SG, Harrison BH (1994) Elution characteristics of gentamicin-PMMA beads after implantation in humans. Orthopedics 17: 415-416.

19. Shyam AK, Sancheti PK, Patel SK, Rocha S, Pradhan C, et al. (2009) Use of antibiotic cement-impregnated intramedullary nail in treatment of infected non-union of long bones. Indian J Orthop 43: 396-402.

20. Buchholz HW, Elson RA, Heinert K (1984) Antibiotic-loaded acrylic cement current concepts. Clin Orthop Relat Res: 96-108.

21. Koo KH, Yang JW, Cho SH, Song HR, Park HB, et al. (2001) Impregnation of vancomycin, gentamicin, and cefotaxime in a cement spacer for two-stage cementless reconstruction in infected total hip arthroplasty. J Arthroplasty 16 : 882-892.
22. Wasko MK, Kaminski R (2015) Custom-Made Antibiotic Cement Nails in Orthopaedic Trauma: Review of Outcomes, New Approaches, and Perspectives. Biomed Res Int 2015: 387186

23. Gallo J, Kolár M, Florschütz AV, Novotný R, Pantůcek R, et al. (2005) In vitro testing of gentamicin-vancomycin loaded bone cement to prevent prosthetic joint infection. Biomed Pap Med Fac Univ Palacky Olomouc Czech Repub 149: $153-158$

24. Penner MJ, Masri BA, Duncan CP (1996) Elution characteristics of vancomycin and tobramycin combined in acrylic bone-cement. J Arthroplasty 11 : 939-944.

25. Kim JW, Cuellar DO, Hao J, Seligson D, Mauffrey C (2014) Custom-made antibiotic cement nails: a comparative study of different fabrication techniques. Injury 45: 1179-1184. 